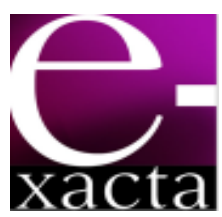

ISSN: 1984-3151

\title{
UTILIZAÇÃo do FRAMEWORK JADE NO DESENVOLVIMENTO DE UM MUSEU VIRTUAL 3D COM RECOMENDAÇÃO Personalizada de Conteúdo
}

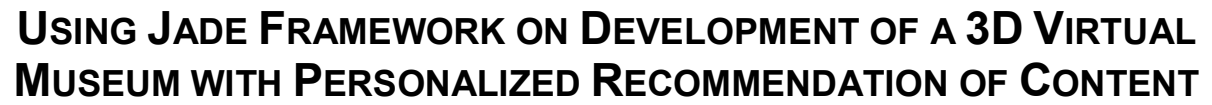

\author{
Íthalo Bruno Grigório de Moura'; Francisco Milton Mendes Neto ${ }^{2 ;}$ Paulo Sérgio Maia de \\ Sousa ${ }^{3}$
}

1 Mestrando pelo Programa de Pós-Graduação em Ciência da Computação (PPgCC) promovido pela Universidade do Estado Do Rio Grande do Norte - UERN e pela Universidade Federal Rural do Semi-Árido - UFERSA Mossoró, RN. Tecnólogo em Análise e Desenvolvimento de Sistemas pelo Instituto Federal do Piauí IFPlithalobgm@gmail.com.

2 Doutor em Engenharia Elétrica. (Processamento de Informação) Universidade Federal de Campina Grande, 2005. Professor adjunto IV da Universidade Federal Rural do Semi-Árido - UFERSA. Mossoró, RN. miltonmendes@ufersa.edu.br.

3 Graduando e pesquisador do Núcleo de Tecnologia em Engenharia de Software - NTES pela Universidade Federal Rural do Semi-Árido - UFERSA Mossoró, RN. paulopsms@gmail.com.

Recebido em: 20/10/2012 - Aprovado em: 09/11/2012 - Disponibilizado em: 30/11/2012

RESUMO: A utilização de ambientes virtuais de aprendizagem em 3D proporciona uma experiência educacional rica em detalhes, sensação de imersão e interação com diversos recursos educacionais. Um museu virtual, a partir de sua plataforma virtual em $3 D$ e dos recursos que esta plataforma pode oferecer, funciona como uma ferramenta educacional eficiente, pois disponibiliza informação aos seus visitantes de forma simples e de fácil compreensão. No entanto, uma limitação do uso de museus virtuais para a aprendizagem é que estes ambientes não levam em consideração as características individuais e contextuais dos visitantes, limitando sua aprendizagem. Assim, este artigo apresenta um museu virtual em 3D denominado Musert, que tem como diferencial a recomendação personalizada de conteúdo. Para isso, utiliza ontologias juntamente com agentes inteligentes para realizar a recomendação personalizada de conteúdo de forma satisfatória.

PalavRas-chave: Museu 3D. Padrão X3D. Browser Xj3D. Recomendação de conteúdo.

ABSTRACT: The use of virtual learning environments in 3D provides an educational experience rich in detail, sense of immersion and interaction with various educational resources. A virtual museum, from its $3 D$ virtual platform and resources that can offer this platform, works as an effective educational tool as it provides information to its visitors in a simple and easy to understand. However, a limitation of the use of virtual museums to learn is that these environments do not take into account the individual and contextual characteristics of visitors, limiting their learning. Thus, this paper presents a 3D virtual museum named Musert, whose differential is personalized content recommendation. It uses ontologies along with intelligent agents to perform personalized content recommendation satisfactorily.

KEYWORDS: 3D museum. X3D standard. Content recommendation. 


\section{INTRODUÇÃO}

A grande disponibilidade de computadores, tanto em escolas como nas residências e a facilidade de acesso a Internet tem influenciado a utilização de recursos tecnológicos com o propósito de promover educação. Nos últimos anos, devido à facilidade de acesso a esses recursos, museus virtuais têm sido cada vez mais utilizados para fins educacionais. Um museu virtual é um sistema complexo, com uma gama de possibilidades para os usuários e por isso, tem mostrado ser eficaz em uma série de projetos de ensino e estão sendo usados para facilitar o processo educacional de maneira diferente da convencional.

A busca por alternativas para suprir a necessidade de preservação do patrimônio histórico e cultural de uma região é cada vez maior, visto que este patrimônio é fundamental para a educação e contribui significativamente na definição da identidade cultural. Entretanto, apesar de sua relevância, uma série de artefatos e documentos de valor histórico imensurável tem sido perdida ao longo do tempo devido à deficiência de recursos e técnicas de preservação que não conseguem evoluir com uma rapidez que evite tais perdas. Isto ocasiona prejuízos irrecuperáveis, provocados por diversos fatores, como tempo, manuseio e armazenamento inadequados (MOURA et. al., 2012). Uma dessas alternativas é a utilização de técnicas de digitalização em três dimensões (3D) em projetos com grande apelo visual. A digitalização em 3D pode ser utilizada para preservar bens do patrimônio histórico e cultural em seus mínimos detalhes, de forma segura, permitindo a construção de réplicas, mesmo quando o original não existe mais, bem como a criação de coleções virtuais acessíveis através da Internet (SOARES et. al., 2009).

Diante disso, é cada vez mais comum a utilização de conteúdo em 3D em Ambientes Virtuais de Aprendizagem (AVA), devido à sua capacidade de promover a sensação de imersão e à disponibilidade de recursos visuais e educacionais. Assim, devido à riqueza de detalhes que as técnicas de digitalização em 3D proporcionam e da grande carga informacional que ambientes virtuais podem oferecer, em muitas situações os usuários são incapazes de identificar as suas reais necessidades de aquisição de informação na presença de uma grande quantidade de dados que Ihes são disponibilizados (ZEB; FASLI, 2011). Na tentativa de resolver este problema, uma das formas encontradas é o armazenamento do perfil dos usuários e das atividades realizadas nos ambientes virtuais, que pode ser feito com o uso de ontologias, e atualização dinâmica destas informações, que pode ser feita por agentes inteligentes. A utilização de ontologias como meio de armazenamento de informações se torna interessante pelo fato de permitir melhor compreensão dos dados por parte de computadores, oferecendo maior precisão dos resultados que são retornados. Além disso, devido à sua forma de representação do conhecimento, a utilização de ontologias permite a comunicação eficiente entre pessoas, agentes de software e sistemas e promove muitas outras vantagens em relação aos mecanismos convencionais de armazenamento de dados (DUNG; FLOREA, 2011; BHATIA; JAIN, 2011).

Com o propósito de superar esses desafios, este artigo apresenta um museu virtual denominado Musert, que utiliza tecnologias como o padrão X3D, o browser Xj3D e técnicas de modelagem específicas para o desenvolvimento do museu e das peças que o compõe. Além disso, mostra como a utilização de agentes de software e ontologias pode promover a recomendação personalizada de conteúdos do acervo, levando em consideração as características do perfil de cada visitante, além de monitorar as atividades do visitante no ambiente virtual.

Assim, este trabalho está dividido em seis seções. A Seção 2 mostra os estudos relacionados e apresenta uma breve comparação entre eles e a proposta 
descrita neste artigo. A Seção 3 traz conceitos relacionados a ambientes virtuais de aprendizagem e a utilização de técnicas de realidade virtual $\mathrm{e}$ aumentada neste tipo de ambiente. A Seção 4 aborda os conceitos e benefícios da utilização de ontologias e agentes inteligentes na recomendação personalizada de conteúdos. A Seção 5 descreve a abordagem proposta neste artigo, que é o desenvolvimento de um museu virtual 3D com recomendação personalizada de conteúdo, e também apresenta as características e as etapas de modelagem e implementação do museu. Já a última seção traz as considerações finas e uma breve discussão sobre trabalhos futuros.

\section{Trabalhos Relacionados}

No trabalho descrito em (SOOKHANAPHIBARN; THAWONMAS, 2009), é relatado o desenvolvimento de um framework para a modularização de um sistema de gerenciamento de conteúdo em museus virtuais no ambiente Second Life (LIU; WANG, 2012). A principal questão é como gerenciar grandes quantidades de informações de um museu e disponibilizar de forma personalizada a informação apropriada para cada visitante deste museu. Com este propósito foi desenvolvido um framework que consiste em dois subsistemas e seis módulos, que são relacionados com o conteúdo a ser apresentado pelo museu e os perfis dos visitantes. As visitas e interação com o museu são o foco do framework, e proporciona a personalização do conteúdo disponibilizado aos usuários durante as visitas pelo museu. Para que o framework desenvolvido obtivesse 0 sucesso desejado, foram adotadas técnicas que já foram utilizadas em sistemas baseados na Web e em sistemas de recomendação. No entanto, estas técnicas ainda precisam ser devidamente ajustadas, e esta tarefa foi deixada como trabalho futuro.

No trabalho apresentado em (FALCÃO; MACHADO, 2010), é descrito um museu virtual imersivo, interativo e itinerante, denominado Museu 31. Este museu tem como principal característica a possibilidade do visitante escolher quais obras deseja visitar. Outro fator importante é a possibilidade de que qualquer pessoa possa ser um curador do museu, sendo necessário apenas o envio de uma peça, modelada tridimensionalmente e que esteja no formato $\mathrm{X} 3 \mathrm{D}$, para o acervo do museu através do browser $\mathrm{Xj} 3 \mathrm{D}$, que já é nativo da aplicação. Além disto, o museu oferece uma interface gráfica em 3D e paralelamente são apresentados menus no qual o visitante poderá selecionar as funcionalidades que mais the interessam.

Desta forma, em comparação aos estudos descritos acima, este trabalho apresentará nas seções seguintes as características de um museu virtual $3 D$ com recomendação personalizada de conteúdo. Para alcançar este objetivo foram utilizadas diversas tecnologias, dentre elas o padrão X3D, o browser Xj3D e a linguagem de programação Java no módulo referente à visualização em três dimensões do museu. Já no módulo de recomendação personalizada de conteúdo, foram utilizados agentes inteligentes, ontologias e técnicas de recomendação de conteúdo. Com base nisso, a utilização do padrão X3D e do browser Xj3D em relação à utilização de mundos virtuais como Second Life, se deve ao fato da fácil interação entre estas tecnologias, juntamente com ontologias e agentes inteligentes para se alcançar o objetivo deste trabalho, que é a recomendação personalizada de conteúdo em ambientes virtuais em 3D. Assim, os detalhes, desafios e resultados alcançados são apresentados nas próximas seções.

\section{Ambientes ViRtuais de Aprendizagem}

AVAs podem ser conceituados como sistemas de software que facilitam os processos de aprendizado individual ou coletivo, utilizando para isso meios eletrônicos. Eles precisam basicamente da internet e 
fornecem muitas funções gerenciais, como, por exemplo, gestão do material educacional, além do acompanhamento e avaliação da aprendizagem dos alunos (BAHIRAEY, 2010). Desta forma, um ambiente virtual pode ser entendido como um espaço virtual que tem o poder de representar uma metáfora do mundo real (DANTAS et. al., 2010).

\subsection{AVAs com Recursos de Realidade VIRTUAL}

A criação de ambientes virtuais 3D, para a representação de AVAs, permite fantasiar sobre infinitas possibilidades para a criação de ambientes que não podem existir fisicamente, ou que, por algum impedimento, não podem disponibilizar todos os recursos desejáveis fisicamente. Com isso, os AVAs 3D permitem 0 surgimento de muitas ideias inovadoras para a construção de personagens (avatares) e desenho arquitetônico de edifícios com fins educacionais. Além disso, a ausência de restrições físicas no desenvolvimento deste tipo de ambiente é bastante significativa em relação às dificuldades encontradas na construção de ambientes reais. Em AVAs 3D não existem as restrições da vida real, como restrições orçamentárias, testes de solo, limitações dos materiais, requisitos de infraestrutura, som ou até mesmo a gravidade. Assim, um simples procedimento 3D pode, por exemplo, transformar e enriquecer cores de paredes envelhecidas e melhorar tristes estilos arquitetônicos (SALEEB; DAFOULAS, 2010).

\subsection{Museus ViRTUAIS}

A popularidade dos ambientes virtuais na web permite o uso deste tipo de sistema para muitas aplicações, dentre elas a educação. Seguindo esta evolução, o desenvolvimento de museus virtuais tem avançado muito nos últimos anos $\mathrm{e}$ isso tem facilitado a disponibilização das peças aos visitantes. Além disso, outro fator de destaque é o poder de representatividade que este tipo de ambiente possui, podendo ocorrer através de diferentes tipos de mídias, tais como texto, imagem, áudio, vídeo, e outros tipos de dados mais complexos, como, por exemplo, objetos que usam técnicas de modelagem em 3D (DANTAS et. al., 2010).

Um museu virtual pode ser caracterizado como uma coleção de artefatos eletrônicos e recursos informativos disponibilizados de forma digital. Uma das vantagens que um museu virtual pode apresentar, em relação aos tradicionais, é a reprodução digital de objetos reais, que ainda existem ou não, oferecendo a possibilidade de observar e interagir com as obras de arte, pertencentes ao museu virtual, que estão localizadas em outro lugar físico (GUIDI et. al., 2010). Outra vantagem é a possibilidade de disponibilizar diversos recursos multimídia, como textos, dados, gráficos e recursos de animação, enriquecendo ainda mais uma visita ao ambiente virtual (CHENGWEI et. al., 2011).

Um museu virtual, a partir da utilização de tecnologias de realidade virtual juntamente com todos os outros recursos, funciona como uma ferramenta educacional eficiente, pois disponibiliza informação aos usuários de forma simples e de fácil compreensão (MOURA et al., 2012a).

Mesmo diante de tantos recursos, pesquisadores concluem que um AVA não pode substituir a interação entre aluno e professor. Um problema que ocorre na maioria dos AVAs é que o conteúdo é passado para todos os alunos da mesma forma e não muda de acordo com as necessidades de cada um (MOURA et. al., 2012).

Assim, nos últimos anos, pesquisadores têm tentado modificar este comportamento passivo e têm apresentado uma série de práticas e tecnologias 
inovadoras para se chegar a uma nova geração de AVAs, onde é possível ter habilidades de interatividade reais e participação no processo de aprendizagem (JUN; BIN, 2011). Desta forma, este trabalho apresenta uma alternativa para que a recomendação de conteúdo seja realizada de forma satisfatória.

\section{Recomendação Personalizada de CONTEÚdo}

Atualmente, há um crescimento exponencial das fontes de dados e este fato torna a aquisição de conhecimento cada vez mais complicada, devido à dificuldade que os usuários têm no momento de identificar as suas reais necessidades de informação (PRIMO; VICARI; SILVA, 2010).

Agentes inteligentes podem realizar diversas tarefas em um AVA, tais como monitorar as atividades do usuário, capturar de forma automática suas informações contextuais, como, por exemplo, a preferência por um determinado tipo de conteúdo e frequência de utilização dos recursos, além de realizar a recomendação personalizada de conteúdo educacional (SILVA; MENDES NETO; JÁCOME JÚNIOR, 2011).

Seguindo esta linha, agentes inteligentes com características pedagógicas (AICPs), além das características de um agente convencional, têm como foco o alcance de objetivos que melhorem o aprendizado dos usuários de AVAs. Devido a essas características, eles têm sido utilizados como tutores, por meio de modelos cognitivos dos usuários, além de proporcionarem um suporte para a aprendizagem personalizada (QU; WANG; JOHNSON, 2004). Atualmente, há um esforço considerável no emprego de AICPs em ambientes tradicionais de aprendizagem. Isto se deve, principalmente, ao potencial destes agentes para proporcionar um aprendizado com maior riqueza de recursos e à exploração das habilidades sociais dos agentes, que podem proporcionar vários cenários de aprendizagem úteis para a colaboração no AVA (SOLIMAN; GUETL, 2010).

Juntamente com agentes de software, ontologias podem ser utilizadas com diversas finalidades em AVAs, sendo uma das suas principais aplicações a personalização, usando para isso, as características específicas do perfil de cada usuário (MIN; WEI; LEI, 2008; PONTES, 2010).

O principal benefício de uma ontologia é a possibilidade de comunicação entre pessoas, agentes e sistemas, já que a ontologia permite o reuso, a representação formal de conceitos e o compartilhamento de conhecimentos (PRADO, 2004).

Devido aos avanços da web semântica e a utilização de ontologias, problemas como armazenamento, organização, compartilhamento e reutilização de informações de forma eficiente podem ser superados. $\mathrm{O}$ uso de ontologias para descrever objetos de aprendizagem permite que diferentes aplicações educacionais compartilhem e reutilizem os mesmos conteúdos educacionais. Além disso, a capacidade de leitura de uma ontologia pelos computadores aumenta a velocidade de consulta às informações compartilhadas e a precisão dos resultados que são retornados (DUNG; FLOREA, 2011).

Com isso, diante das vantagens que as ontologias possuem, neste trabalho foram utilizadas ontologias para armazenar todas as informações dos perfis dos usuários, as descrições das peças a serem recomendadas e as informações que influenciam dinamicamente o comportamento da aplicação.

\section{MuserT}

Nesta seção são apresentadas as principais características do museu virtual proposto neste artigo. 
A seguir é detalhada a arquitetura da proposta juntamente com as técnicas e tecnologias que, juntas, viabilizam a recomendação personalizada de conteúdo.

\subsection{ARQUITETURA}

Este trabalho tem como diferencial a personalização de conteúdo em um museu virtual denominado Musert, tendo como base para isso, ontologias e AICPs. A ideia principal desta abordagem é identificar os requisitos do usuário, ou seja, suas preferências e suas características, e criar um modelo deste usuário. Nesse modelo deve constar o seu conhecimento expresso por meio de um conjunto de termos pertencentes a uma ontologia comum, possibilitando adaptar o conteúdo de forma individual.
A arquitetura desta proposta pode ser visualizada na Figura 1, onde são representados os AIPCs, ontologias e a interação dos usuários com o museu.

Como pode ser visto na Figura 1, é possível ter uma perspectiva geral dos processos de funcionamento do museu. Assim, inicialmente há a autenticação do visitante junto ao museu. Após a autenticação, a visualização das descrições das peças é acionada a partir da aproximação do visitante aos sensores das peças, que ficam localizados em locais prédeterminados do museu. Antes disto, no entanto, o visitante precisa realizar um cadastro para que suas características pessoais sejam armazenadas na ontologia de contexto estático, que contém informações como, por exemplo, nome, idade e escolaridade.

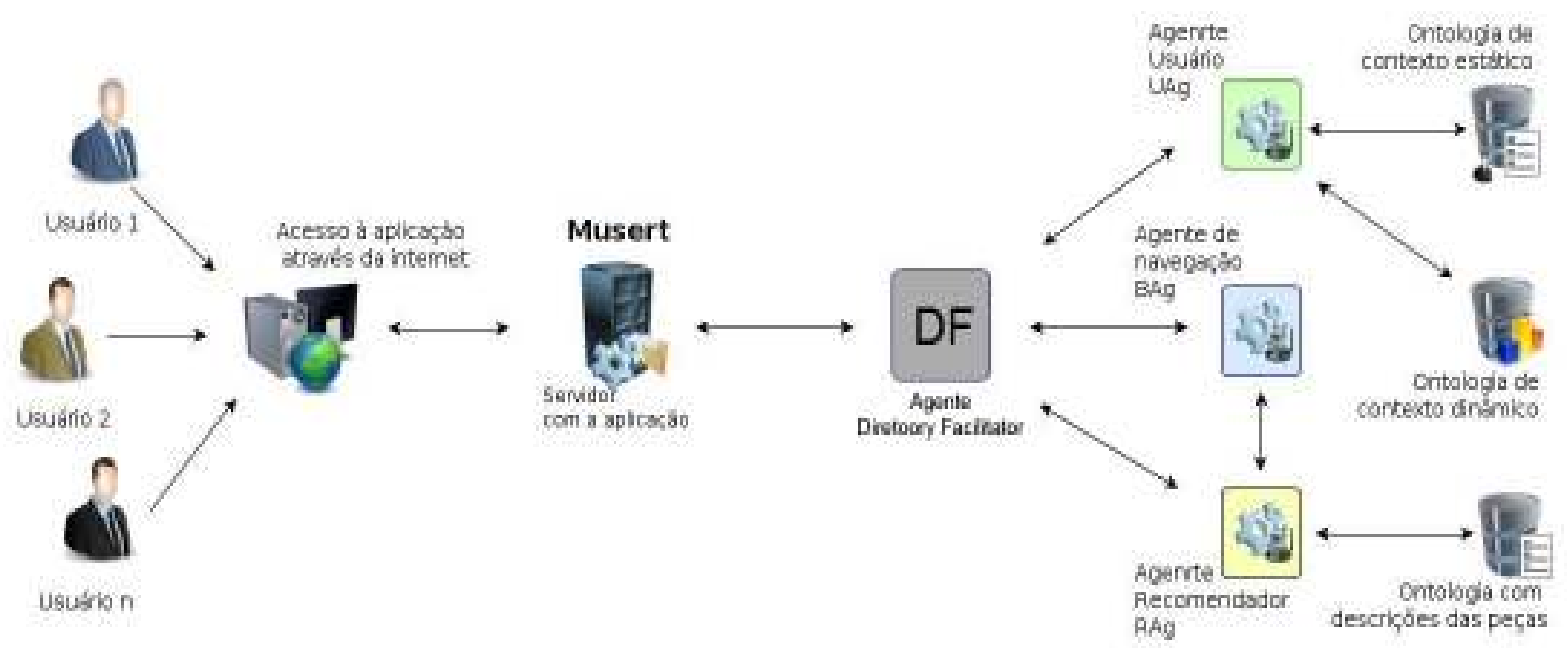

Figura 1 - Arquitetura do museu virtual proposto e com a utilização de agentes de software e ontologias na disponibilização personalizada de conteúdo.

Além desta ontologia, há a ontologia de contexto dinâmico, que é responsável por armazenar informações como quantidade de visitas, peças visitadas, dentre outras informações de caráter dinâmico, que representam a interação do visitante com o ambiente virtual.

\subsection{Agentes DE SOFTWARE COM Características Pedagógicas}

$\mathrm{Na}$ abordagem proposta foram implementados quatro agentes: Agente de Navegação (BAg - Browsing Agent), Agente Usuário (UAg - User Agent), Agente Recomendador (RAg - Recommender Agent) e o agente DF (Diretory Facilitator). Cada agente tem um 
objetivo especifico, mas se relaciona com os demais para alcançar o objetivo principal, que é a recomendação personalizada de conteúdo.

O UAg é responsável por monitorar as atividades dos visitantes e recuperar, as ontologias de contexto estático e dinâmico, as preferências de conteúdo que compõem os perfis dos visitantes e os seus respectivos históricos de peças visitadas. Com base no histórico de visitas, o UAg pode verificar o perfil de outros visitantes que possuem, em seus históricos, preferências similares. Os UAgs também capturam as informações do contexto dinâmico do estudante. Para isso, o UAg realiza sua ação no momento em que o estudante se autentica na aplicação. Em seguida, todas essas informações são cadastradas no agente DF.

O RAg tem como propósito detectar a descrição das peças que são adequadas ao perfil do estudante, de acordo com as informações providas pelo Agente DF e aquelas quanto ao acervo do museu, obtidas da ontologia de descrições das peças. Assim, o RAg encontra, inicialmente, a descrição que seria mais adequada de acordo com o perfil do visitante. Em seguida, com base nessas informações, o RAg verifica a quantidade de visitas que o usuário fez àquela peça juntamente com a quantidade de visitas que ele fez ao museu, e cadastra estas informações no agente DF, que serão consultadas pelo BAg.

O BAg interage diretamente com os sensores de proximidade presentes por todo o museu, principalmente nas peças. A partir da aproximação de um visitante a uma peça, o sensor percebe a intenção do visitante em obter informações sobre ela. Após isso, o BAg gerencia a disponibilização do conteúdo juntamente com os outros agentes.

O agente DF provê, para outros agentes, o serviço de páginas amarelas. Esse serviço consiste em uma lista de todos os agentes e os respectivos serviços oferecidos por eles. Todo agente que desejar publicar seus serviços para outros agentes deve encontrar um agente DF apropriado e requisitar o registro de sua descrição de serviço. Além disso, os agentes podem retirar e modificar o seu próprio registro de um DF e buscar neste, de acordo com um critério de busca, por um registro de serviço fornecido por outro agente. Esse componente é de extrema importância para o Sistema Multiagente (SMA) proposto. Outro fator a ser observado é que o bom desempenho do RAg depende diretamente de um mecanismo eficiente para a representação do conhecimento. Assim, o mecanismo de recomendação desenvolvido considera as informações do perfil do visitante, como, por exemplo, idade, escolaridade, conhecimento sobre o tema do museu, dentre outras, contidas na ontologia de contexto estático, além da quantidade de visitas e as últimas peças visitadas, as quais estão contidas na ontologia de contexto dinâmico. Estas informações são ponderadas de acordo com as descrições disponíveis para cada peça e são utilizadas também para sugerir a ordem da visita e que peças devem ser visitadas, levando em consideração as atividades de usuários com perfis semelhantes. Com base nisto, objetiva-se alcançar uma recomendação eficiente do conteúdo descritivo das peças do museu.

\subsubsection{Modelagem dos Agentes de Software}

Com o surgimento do paradigma de Programação Orientada a Agentes, várias metodologias para a modelagem de SMAs foram propostas nos últimos anos. Analisando as vantagens e desvantagens de cada uma delas, a metodologia escolhida para modelagem dos agentes deste trabalho foi a MASCommonKADS+, proposta em (MORAIS II, 2010), que consiste em uma extensão à MAS-CommonKADS Para uma melhor compreensão dos benefícios da utilização da MAS-CommonKADS+, é interessante, inicialmente, entender em que consiste a MASCommonKADS, que se trata de uma metodologia_de Engenharia de Software Orientada a Agentes que 
estende, com uma abordagem que guia o processo de análise e projeto de SMAs a CommonKADS, que é a principal metodologia estruturada de suporte à engenharia do conhecimento. A MAS-CommonKADS utiliza técnicas de modelagem bem conhecidas, tais como diagramas de caso de uso e diagramas de sequência de mensagens. Entretanto, adota novas perspectivas dirigidas pela metáfora dos agentes (MORAIS II, 2010).

A metodologia MAS-CommonKADS+ mantém muitos aspectos dos modelos já propostos na metodologia MAS-CommonKADS, porém realiza algumas modificações e adiciona novos conceitos. Desta forma, foram adicionados os modelos de requisitos, de papéis e de recursos, enquanto que os modelos de organização, de interação e de projeto foram alterados, com o intuito de complementar a especificação dos diagramas da AML (Agent Modeling Language).

Seguindo os passos da metodologia MASCommonKADS+, essa seção apresenta a modelagem do SMA desenvolvido neste trabalho. Assim, é representado cada modelo através de um diagrama, contribuindo assim para a compreensão do SMA. Desta forma, o modelo de tarefas continua sendo utilizado conforme especificado na metodologia MASCommonKADS e descreve as tarefas com as quais os agentes se preocupam e os objetivos. Abaixo é apresentado o modelo de tarefas do SMA proposto.

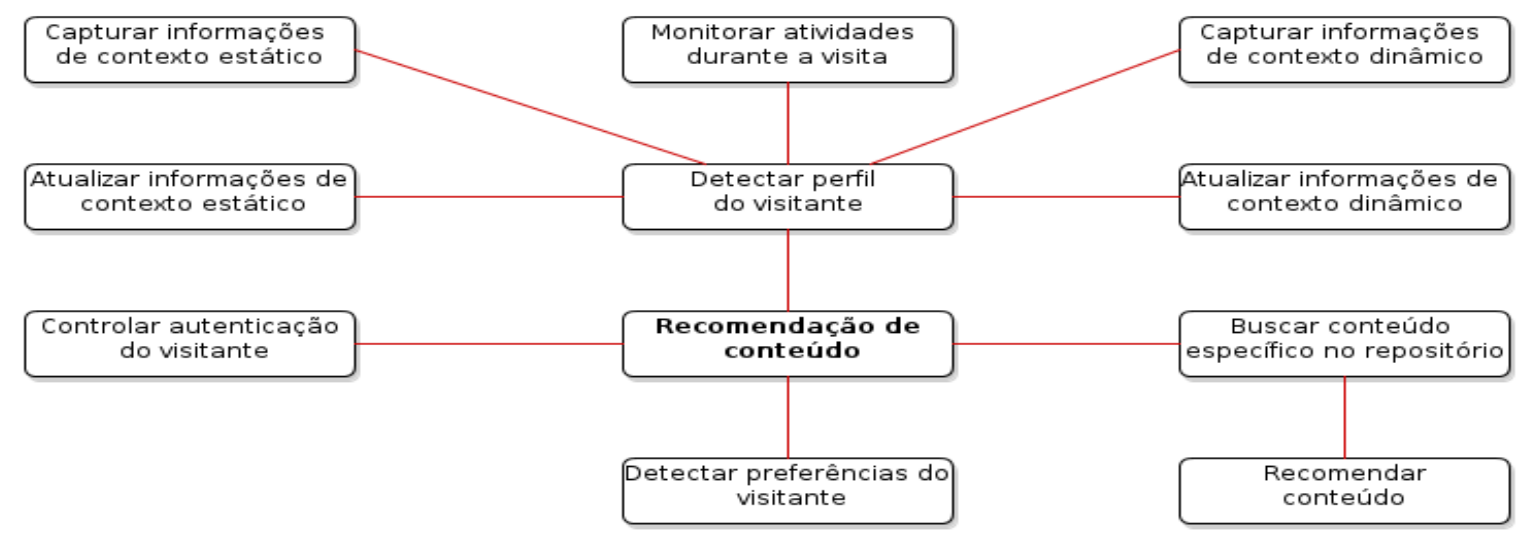

Figura 2 - Modelo de Tarefas.

A tarefa principal deste SMA é a recomendação de conteúdo referente à descrição das peças expostas no museu. Essa tarefa, por sua vez, foi decomposta em outras subtarefas, que têm como intuito final auxiliarem no cumprimento da tarefa principal. O próximo passo consiste em definir os papéis que cada agente irá realizar no sistema, visto que um único agente pode ser responsável por vários papéis em um SMA.
Já o modelo de papéis tem como objetivo a identificação dos papéis do sistema e a representação das atividades descritas no modelo de tarefas. Um papel é uma abstração que define as tarefas que um agente deve realizar dentro de uma organização. Um único agente pode ser responsável por vários papéis em um sistema. Logo, a inclusão deste modelo é de extrema relevância, pois permite modelar os vários papéis, que um agente pode exercer. 
Na Figura 3 é possivel perceber que foram definidos três tipos de papéis para atender às tarefas inerentes ao Modelo de Tarefas: visitante, recomendador e navegação.

\begin{tabular}{|c|}
\hline $\begin{array}{c}\text { 《<entity role»> } \\
\text { Navegaçåo }\end{array}$ \\
\hline 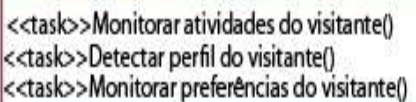 \\
\hline
\end{tabular}

Figura 3 - Modelo de Papéis.
Apesar de ser possível que cada agente possa realizar mais de um papel, para simplificar o entendimento e distinguir bem as atividades de cada agente, decidiu-se de que cada um desses papéis será realizado por um agente. Uma vez construído o Modelo de Papéis, deve ser desenvolvido o Modelo de Organização, que serve para descrever a estrutura organizacional dos papéis no sistema, ou seja, como os papéis estão relacionados entre si. A Figura 4 mostra o Modelo de Organização correspondente aos papéis exibidos na Figura 3.

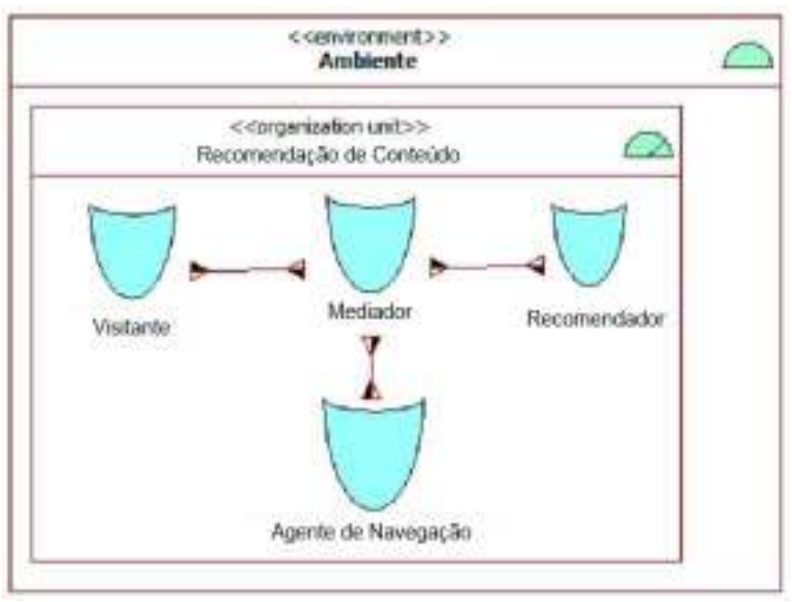

Figura 4 - Modelo de Organização.

Nesse modelo é possível perceber a presença de outro papel, o mediador. O mediador permitirá que os agentes se comuniquem sem que, inicialmente, eles se conheçam. O motivo dele não fazer parte do Modelo de Papéis é que o mesmo não realiza tarefas que compõem a finalidade principal do SMA, ou seja, não realiza uma das tarefas que compõem o Modelo de Tarefas. Mesmo assim, essa tarefa é de extrema relevância, pois é através dela que os agentes conseguem informações a respeito dos serviços de outros agentes e conseguem se comunicar uns com os outros. Ou seja, ele funciona como um agente intermediário, permitindo que os agentes cadastrem informações e que pesquisem por informações de outros agentes. O papel do mediador será realizado pelo agente Directory Facilitator (DF), o qual é provido pela própria plataforma JADE. É necessário apenas codificar a forma como os agentes criados no SMA irão se comunicar com o DF. Além destes detalhes, é necessário que se tenha uma visão do comportamento dinâmico do SMA, o que é possível conseguir através do Modelo de Interação. O modelo de interação consiste na junção dos modelos de coordenação e de comunicação da MAS-CommonKADS. Nele são descritas, através da AML, todas as interações entre os agentes. Cada interação deve obedecer a um protocolo de interação, o qual estabelece como os agentes podem se comunicar. A Figura 5 mostra o

e-xacta, Belo Horizonte, v. 5, n. 2, p. 83-97. (2012). Editora UniBH Disponível em: www.unibh.br/revistas/exacta/ 
modelo de interação entre os agentes que compõem o

SMA proposto.

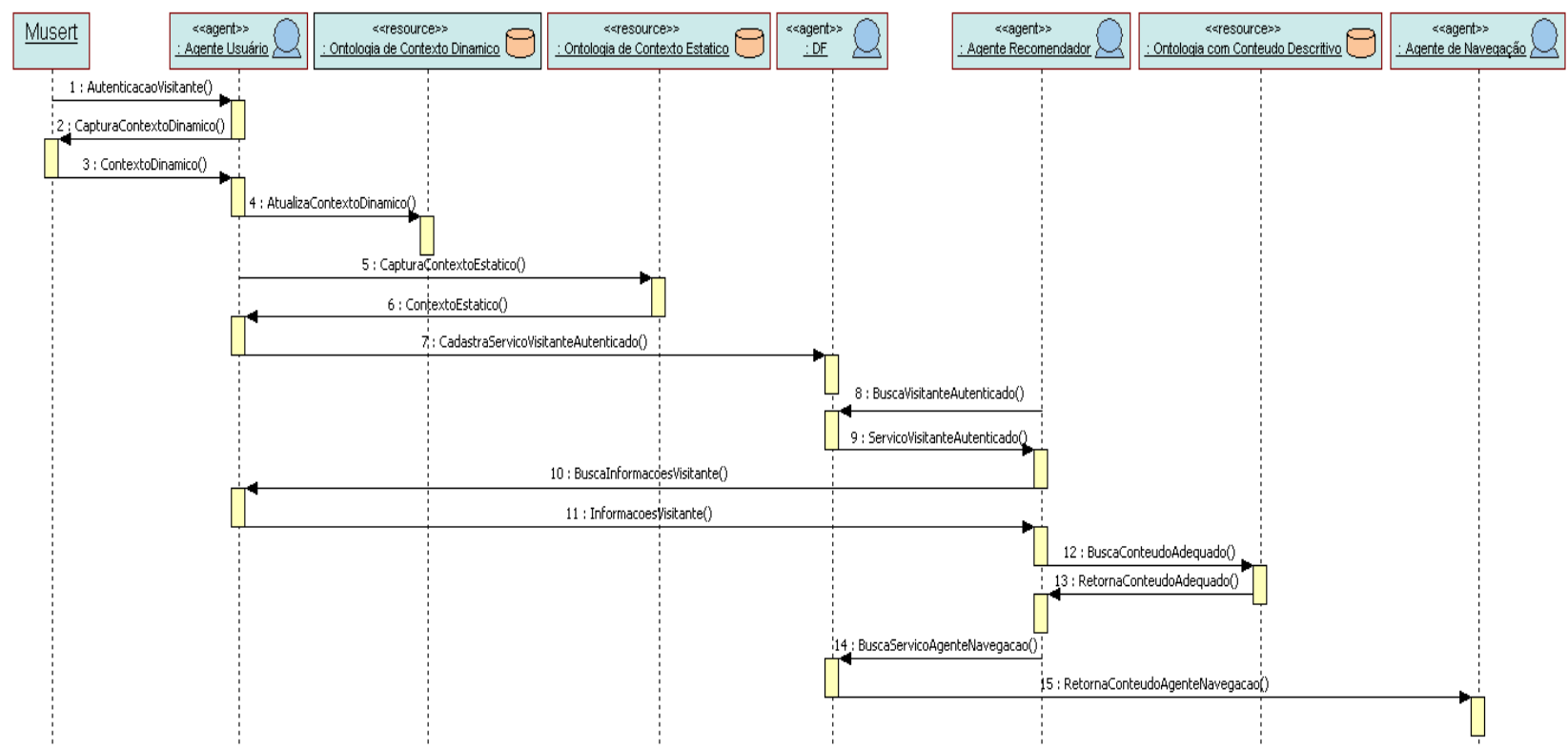

Figura 5 - Arquitetura do museu virtual proposto e com a utilização de agentes de software e ontologias na disponibilização personalizada de conteúdo.

No modelo apresentado na Figura 5, é possível visualizar toda a interação que ocorre entre os agentes e os recursos que são consultados pelos quatro agentes que compõem o sistema. O diagrama mostra desde o comportamento do UAg no momento em que o visitante se autentica no Musert até a informação chegar ao BAg, que irá buscar pelo conteúdo que mais se adéqua ao perfil do visitante.

Também é possível perceber que as informações de contexto estático e dinâmico do estudante precisarão ser armazenadas. Uma forma possível de se armazenar essa informação seria com a utilização de um banco de dados. Porém, o uso de uma ontologia para a representação desse conhecimento mostra-se como uma alternativa mais útil, visto que esse conhecimento pode ser utilizado por outras partes da aplicação e, caso seja necessário modificar o comportamento desta, pode-se criar uma nova forma de representar a ontologia, sem modificar a codificação em si. Assim, é por este motivo que estão sendo utilizadas duas ontologias, sendo uma para representação do contexto estático e outra para representação do contexto dinâmico do estudante.

\subsubsection{Comunicação, Gerenciamento dos Agentes de Software}

$\mathrm{Na}$ comunicação e gerenciamento dos agentes, foi utilizado o framework JADE (Java Agent Development Framework), que é voltado para o desenvolvimento de sistemas multiagente e tem como uma de suas características, a sua total implementação em linguagem Java e fornece um conjunto de recursos técnicos para 0 desenvolvimento de sistemas multiagente. Além disso, provê uma plataforma de agentes distribuídos, gestão facilitada dos agentes e comunicação entre agentes com troca de mensagens. A principal vantagem em utilizar o JADE reside no fato de que é possível utilizar todos os componentes da especificação FIPA, (Foundation for Intelligent Physical Agents), visto que o JADE atende todas as especificações deste padrão e ainda o estende, sendo 
que um desses componentes é o agente DF (FIPA, 2012). Outra vantagem dessa plataforma consiste na possibilidade de consultar e utilizar, através do MTS (Message Transport Service), os agentes DFs que estejam sendo executados em outras plataformas de agentes. Assim, o DF utilizado na plataforma JADE deste trabalho pode, por exemplo, ser consultado, por meio da internet, por um agente que esteja sendo executado em outro local, através do protocolo MTP
(Message Transport Protocol). Essa característica permite a criação de uma rede de colaboração entre os agentes, mesmo que estejam localizados, por exemplo, em universidades distintas. A Figura 6 mostra, através da interface gráfica do JADE, como ocorre a comunicação entre os agentes descrita através dos modelos textuais dos agentes apresentados anteriormente.

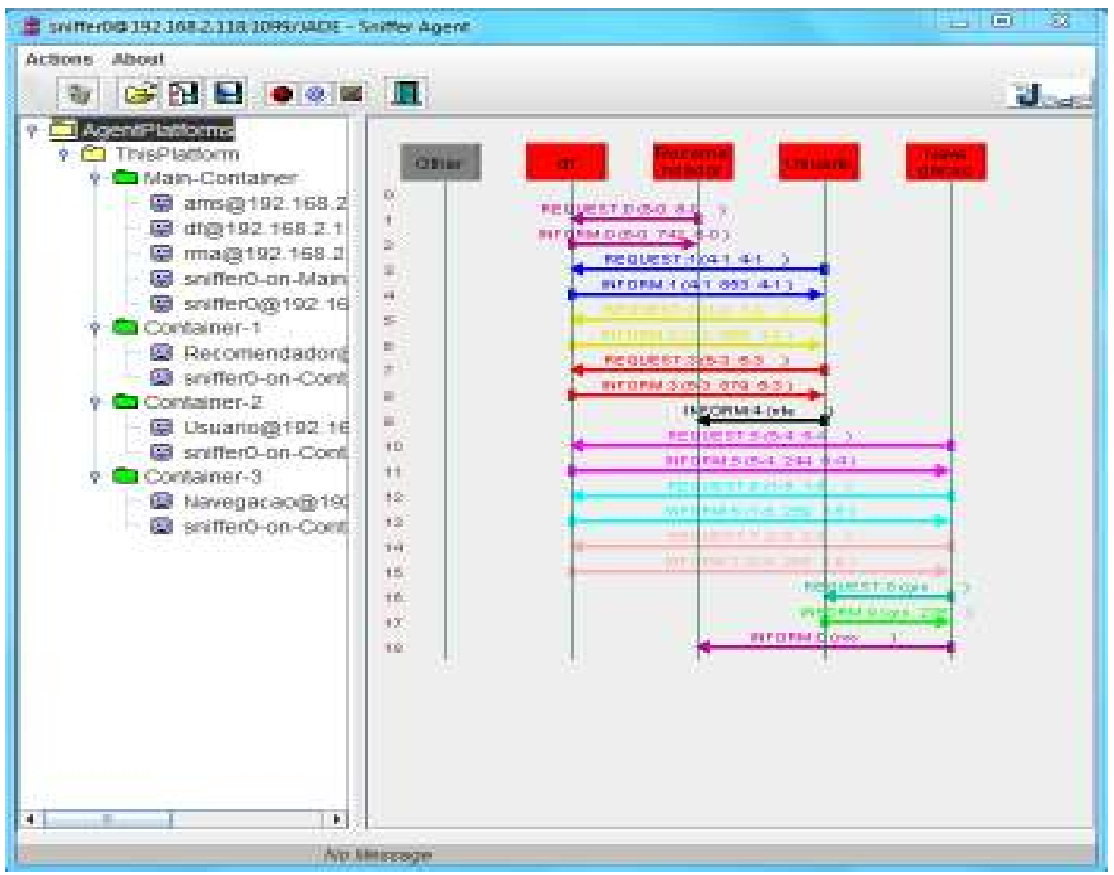

Figura 6 - Comunicação entre agentes através do JADE Sniffer Agent.

Como pode ser visto na Figura 6, inicialmente, o RAg solicita por informações de serviço ao agente DF. Em seguida, o DF responde com as informações de serviços disponíveis. Desta forma, a comunicação entre os três agentes e o agente DF é mantida durante toda a utilização do museu virtual.

\subsection{Aspectos da Implementação do Museu Virtual Proposto}

Para o desenvolvimento do AVA proposto foram estudadas diversas ferramentas e tecnologias. $O$ objetivo principal era permitir a navegação no ambiente virtual, bem como permitir a interação com seus diversos elementos de forma personalizada, utilizando para isso ontologias e agentes de software. Foram consideradas diversas tecnologias e mundos virtuais, como o Second Life (LIU; WANG, 2012) e o OpenSim (RIDGEWELL et. al., 2010), pela facilidade de tratamento gráfico, navegabilidade e interatividade. Entretanto, além da necessidade de disponibilização do conteúdo na Web, a recomendação de conteúdo de forma personalizada exige a utilização de tecnologias específicas e, por esta razão, para este 
trabalho foram escolhidos o padrão X3D e o browser Xj3D. O X3D (Extensible 3D) é um padrão adotado internacionalmente para 3D na Web. Ele é utilizado para construir ambientes virtuais tridimensionais complexos. Trata-se de um padrão aberto que permite descrever em um arquivo formas e comportamentos de um ambiente virtual. As formas são descritas por figuras geométricas e os comportamentos da cena podem ser controlados internamente pelo arquivo X3D e externamente por linguagens de programação ou script. A escolha do X3D como ferramenta de implementação deveu-se a facilidade de utilização com a linguagem de programação Java juntamente com a facilidade de comunicação com o browser Xj3D. Além do fato do X3D oferecer suporte a diversas mídias e formas de interação, inclusive contendo APIs que adicionam diversas funcionalidades às já existentes (MEDEIROS; MACHADO, 2010).

Browsers X3D consistem em aplicações capazes de interpretar e processar as cenas, que na verdade são arquivos X3D, apresentando os modelos tridimensionais, animados ou não, e permitindo interações do usuário com os objetos do cenário. A escolha do browser deve ser feita de acordo com as necessidades do AVA a ser construído. Com isso, foi escolhido o browser $X j 3 \mathrm{D}$, que se trata de um software de código aberto e que possibilita a integração do ambiente com scripts na linguagem de programação Java, que é a linguagem utilizada na implementação dos agentes e na comunicação destes com as ontologias (FALCÃO; MACHADO, 2010) (MEDEIROS; MACHADO, 2010).

\subsubsection{MODELAgEM TRIDIMENSIONAL}

Para o desenvolvimento do Musert foram definidas três etapas:

- a modelagem tridimensional do museu, denominada de etapa de modelagem,
- o desenvolvimento da interface gráfica a ser utilizada pelo visitante, que é a etapa de interface e, por último,

- a implementação dos agentes e ontologias, que são os reais responsáveis pela recomendação personalizada de conteúdo, denominada etapa de recomendação de conteúdo.

A etapa de modelagem foi concebida como primeira atividade a ser executada. Para isso foi utilizado o Blender. Trata-se de um software livre e de código aberto para modelagem 3D, que possui exportador para o formato $\mathrm{X} 3 \mathrm{D}$, amplamente utilizado pela comunidade de desenvolvedores de aplicações 3D (MOURA et. al., 2012). O resultado da utilização desta ferramenta pode ser visualizado na Figura 7 , onde é apresentada uma peça 3D em fase de modelagem.

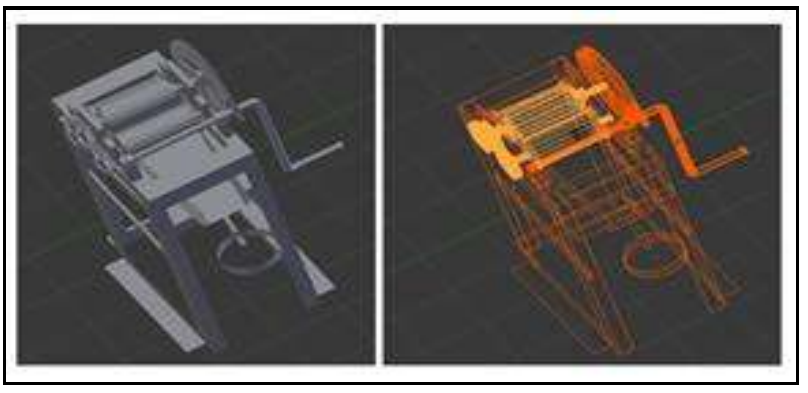

Figura 7 - Peça 3D do Musert em fase de modelagem com utilização da ferramenta Blender.

Como o museu será disponibilizado na Internet, a modelagem foi otimizada em um nível que a qualidade gráfica do ambiente virtual não inviabilizasse o uso devido à quantidade de informações trocadas entre o servidor onde a aplicação se encontra e seus visitantes. Deste modo, foram utilizadas texturas otimizadas em relação à quantidade de dados, mas que ao mesmo tempo promovem uma aparência realista ao ambiente. Adicionalmente foram utilizadas técnicas de implementação peculiares do X3D, que evitam a replicação desnecessária de código e, consequentemente, aumentam a velocidade de 
transmissão das informações oferecidas pelo ambiente (MOURA et. al., 2012a).

No projeto da interface foi utilizada a linguagem de programação Java juntamente com o browser Xj3D. O Xj3D é um browser em conformidade com as especificações do X3D, implementando, inclusive, funcionalidades não especificadas pelo padrão, o que facilita o desenvolvimento de ambientes virtuais 3D com interatividade complexa e alta qualidade gráfica. No Musert, o visitante terá acesso ao museu e poderá navegar por todas as salas do museu e visitar as peças contidas em cada sala. A utilização de sensores e o monitoramento dos agentes permite a interação com as peças de forma inteligente, ou seja, fornecendo uma descrição das peças de forma personalizada. $O$ resultado da modelagem do museu pode ser visualizado na Figura 8, onde é mostrada a tela inicial apresentada aos visitantes.

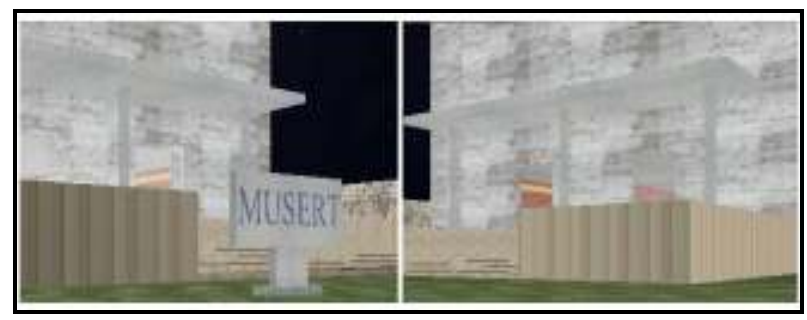

Figura 8 - Visualização externa do Museu.

No Musert, a recomendação de conteúdo é provida pela interação entre os agentes e as ontologias com as descrições das peças e com as informações dos visitantes. A ontologia de contexto estático contém informações cadastrais básicas de cada visitante, enquanto que a ontologia de contexto dinâmico contém as informações referentes à interação do visitante com o museu, ou seja, nesta ontologia são armazenadas informações como as peças que já foram visitadas, a quantidade de visitas ao museu, a quantidade de visitas a cada peça e as salas visitadas.

Com isto, por meio da inferência realizada pelos agentes nestas duas ontologias, é possível recomendar uma rota de visita e uma descrição mais apropriada das peças para cada perfil de usuário. Esta recomendação proporciona uma experiência educacional ímpar e, ao mesmo tempo, eficaz para a necessidade de cada visitante.

\subsubsection{Utilização de Sensores}

A utilização de sensores foi adotada com o objetivo de permitir a geração de eventos de acordo com a ação do visitante no museu. Um exemplo de sensor utilizado é o ProximitySensor. Este sensor gera eventos ao chegar próximo de um determinado objeto, neste caso, peças do museu e pontos específicos que acionam mecanismos para maximizar a experiência educacional do visitante do museu. Esses sensores estão sendo utilizados para que o visitante possa visualizar as informações a respeito das peças de forma interativa. Assim, eles estão sendo empregados para ativar a exibição das descrições das peças, para recomendar salas a serem visitadas e rotas a serem adotadas, além de informações sobre o próprio museu (MOURA et. al., 2012b). Na Figura 9 é possível observar a interação do visitante com o museu a partir da aproximação de uma região específica do museu.

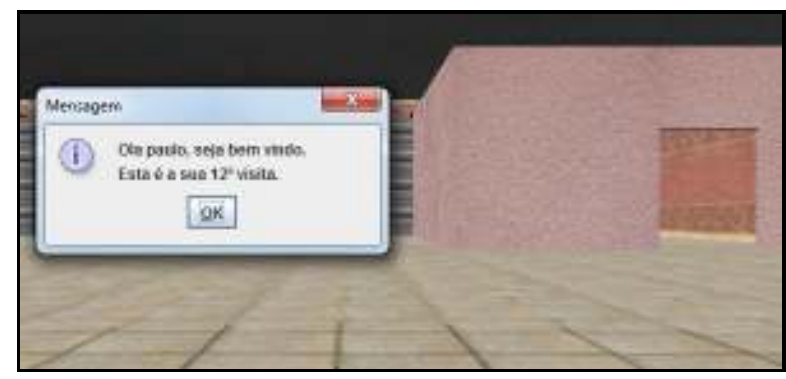

Figura 9 - Interação entre visitante e o museu a partir da atuação de agentes de software.

Como pode ser visto na Figura 9, há sensores de proximidades nas peças e em pontos específicos do museu, com o propósito de promover uma experiência educacional diferente por meio de sugestão de salas a serem visitadas e a ordem em que elas são visitadas.

e-xacta, Belo Horizonte, v. 5, n. 2, p. 83-97. (2012). Editora UniBH. Disponível em: www.unibh.br/revistas/exacta/ 


\section{CONCLUSÕES}

Neste artigo, foi descrita a implementação de um museu virtual 3D, que utilizou tecnologias como a ferramenta de modelagem Blender para a modelagem do museu e das peças que o compõem, além do padrão X3D e do browser Xj3D para disponibilizar o conteúdo do museu na Web. Além disso, também foram utilizados a linguagem de programação Java, agentes inteligentes e ontologias para a realização da recomendação inteligente de conteúdos do acervo. Desta forma, a solução proposta objetiva tornar a aprendizagem, a partir das visitas ao museu, adequada às necessidades de cada visitante.

Desta forma, como trabalhos futuros, pretende-se submeter o ambiente desenvolvido à avaliação de um museólogo, para que a aplicação tenha melhor abordagem no que tange ao aspecto de conteúdo descritivo das peças recomendadas. Tem-se também como proposta futura a integração do sistema com redes sociais ou outros mecanismos de identificação para ajudar no levantamento do perfil do usuário e melhorar a forma de coleta de dados, evitando o preenchimento de formulários antes de utilizar o ambiente. Além disso, objetiva-se mensurar o quanto a abordagem é eficaz ao realizar a recomendação sob a ótica educacional, tendo em vista que testes preliminares mostram que o ambiente realiza essa operação de forma satisfatória, obedecendo a todos os critérios apresentados anteriormente.

Com base nisso, objetiva-se realizar um estudo de caso com uma turma de um curso de ensino a distância para verificar o impacto da abordagem proposta na adequação do conteúdo.

\section{REFERÊNCIAS}

BAHIRAEY, M. H., Quality of collaborative and individual learning in virtual learning environments. In: SECOND INTERNATIONAL CONFERENCE ON E-LEARNING AND E-TEACHING (ICELET), p. 33-39, 2010.

BHATIA, C. S.; JAIN, S. Semantic Web Mining: Using Ontology Learning and Grammatical Rule Inference Technique. In: INTERNATIONAL CONFERENCE ON PROCESS AUTOMATION, CONTROL AND COMPUTING (PACC), p. 1-6., 2011.

CHENGWEI, Y.; CHENGLE, Y.; SHIJUN, L.; XIANGXU, M.; RUI, W. An Approach of Personalized 3D Scene Customization Based on Multimedia Resource. In: INTERNATIONAL CONFERENCE ON MULTIMEDIA AND SIGNAL PROCESSING (CMSP), p. 131-135, 2011.

DANTAS, R. R.; MELO, J. C. P.; LESSA, J.; SHNEIDER, C.; TEODÓSIO, H.; GONÇALVAES, L. M. G. A Path Editor for Virtual Museum Guides. In: IEEE INTERNATIONAL CONFERENCE ON VIRTUAL ENVIRONMENTS HUMAN-COMPUTER INTERFACES AND MEASUREMENT SYSTEMS (VECIMS), 2010.
DUNG, P. Q. FlOREA, A. M. An Architecture and a;Domain Ontology for Personalized Multi-agent eLearning Systems. In: THIRD INTERNATIONAL CONFERENCE ON KNOWLEDGE AND SYSTEMS ENGINEERING (KSE), p. 181-185, 2011.

FALCÃO, E. L.; MACHADO L. S. Museu 3l: Publicação e Visitação Online de Acervos Tridimensionais. In: VII WORKSHOP DE REALIDADE VIRTUAL E AUMENTADA (WRVA), São Paulo, SP, 2010.

FIPA. Welcome to the Foundation for Intelligent Physical Agents. Site Oficial do Padrão FIPA, 2011. Disponivel em: <http://www.fipa.org/>. Acesso em: out. 2012.

GUIDI, G.; TROCCHIANESI, R.; PILS, G.; MORLANDO, G. e SEASSARO, A. A Virtual Museum for Design: New forms of interactive fruition. In: 16TH INTERNATIONAL CONFERENCE ON VIRTUAL SYSTEMS AND MULTIMEDIA (VSMM). p. 242-249, 2010

JADE. Java development framework: an opensource platform for peer-to-peer agent based applications. Disponível em: <http://jade.tilab.com> Acesso em: set. 2012. 
JUN, J. Z.; BIN, W. Z. Ideas transforming in the public arts education of virtual museum. In: $6 \mathrm{TH}$ INTERNATIONAL CONFERENCE ON COMPUTER SCIENCE \& EDUCATION (ICCSE), p. 649-653, 2011.

LIU, C. J.; WANG, G. Using Second Life in ESL/EFL Teaching and Reacher-Training in the Web2.0 Environment. In: IEEE SYMPOSIUM ON ELETRICAL \& ELETRONICS ENGINEERING (EEESYM), p. 717720, 2012.

MEDEIROS, D. P. S.; MACHADO, L.S. X3D e Integração Multimídia para Representação de um Sítio Arqueológico. In: VII WORKSHOP DE REALIDADE VIRTUAL E AUMENTADA (WRVA), São Paulo, SP, 2010.

MIN, W.; WEI, C.; LEI, C. Research of ontologybased adaptive learning system. In: INTERNATIONAL SYMPOSIUM ON COMPUTATIONAL INTELLIGENCE AND DESIGN, ISCID '08, v. 2 , p. $366-370$., 2008.

MORAIS II, M. J. D. O. MAS-CommonKADS+: Uma Extensão à Metodologia Mas-CommonKADS para Suporte ao Processo Detalhado de Sitemas Multiagentes Racionais. Dissertação (Mestrado)Universidade Estadual do Ceará - UECE. Fortaleza, CE. 2010.

MOURA, Í. B. G.; LIMA, J. D.; SOUSA, P. S. M.; MENDES NETO, F. M. Musert: Um Museu Virtual em 3D com Recomendação Personalizada de Conteúdo. In: XXIII SIMPÓSIO BRASILEIRO DE INFORMÁTICA NA EDUCAÇÃO, SBIE. Rio de Janeiro, RJ, 2012.

MOURA, Í. B. G.; LIMA, J. D; MENDES NETO, F. M.; SOUSA, P. S. M., Musert: Um Museu Virtual em 3D para a Preservação do Patrimônio Histórico e Cultural do Semiárido Brasileiro. In: V ESCOLA REGIONAL DE COMPUTAÇÃO DOS ESTADOS DO CEARÁ, MARANHÃO E PIAUÍ (ERCEMAPI). 2012.

MOURA, Í. B. G.; MENDES NETO, F. M.; SOUSA, P. S. M.; LIMA, J. D. Utilização do Padrão X3D no Desenvolvimento de um Museu Virtual Imersivo com Recomendação Personalizada de Conteúdo Através do Uso de Agentes Inteligentes e Ontologias. In: IV WORKSHOP DE REALIDADE VIRTUAL E AUMENTADA (WRVA), Paranvaí, PR, 2012.

PRIMO, T. T.; VICARI, R. M.; SILVA, J. M. C. Rumo ao Uso de Metadados Educacionais em Sistemas de Recomendação. In: XXI SIMPÓSIO BRASILEIRO DE INFORMÁTICA NA EDUCAÇÃO, SBIE, João Pessoa, PB, 2010.
PONTES, A. A. Uma Arquitetura de Agentes para Suporte a Colaboração na Aprendizagem Baseada em Problemas em Ambientes Virtuais de Aprendizagem. Dissertação (Mestrado)Universidade Federal Rural do Semiárido - UFERSA, Mossoró-RN, 2010.

PRADO, S. Um experimento no uso de ontologias para reforço da aprendizagem em educação à distância. Tese (Doutorado em Engenharia)-Escola Politécnica, Universidade de São Paulo. São Paulo, 2004.

QU, L.; WANG, N.; JOHNSON, W. L., Choosing When to Interact with Learners. In: Proceedings of the 9th international Conference on Intelligent User Interfaces, 2004.

RIDGEWELL, W., KUMAR, V., LIN, O.; KINSHUK. OpenSim Virtual as Platform for Enhanced Learning Concepts. In: IEEE INTERNATIONAL CONFERENCE ON ADVANCED TECHNOLOGIES (ICALT). p.623-624, 2011.

SALEEB, N.; DAFOULAS, G. Pedagogical immigration to 3D virtual worlds: A critical review of underlying themes and their concepts. In: INTERNATIONAL CONFERENCE ON INFORMATION SOCIETY (I-SOCIETY), p. 401-409, 2010.

SILVA, C. N.; MENDES NETO, F. M.; JÁCOME JÚNIOR, L. MobiLE: Um ambiente Multiagente de Aprendizagem Móvel para Apoiar a Recomendação Sensível ao Contexto de Objetos de Aprendizagem. Artigo Completo, In: XXII SIMPÓsIO BRASILEIRO DE INFORMÁTICA NA EDUCAÇÃO, SBIE. Aracaju, SE, 2011.

SOARES, I. J. A.; SILVA, L.; BELLON, O. R. P.; VRUBEL, A., 3D Virtual Museum for Digital TV. In: WebMedia '09, Fortaleza, CE, 2009.

SOLIMAN, E.; GUETL, C. Intelligent Pedagogical Agents in immersive virtual learning environments: A review. In: PROCEEDINGS OF THE 33RD INTERNATIONAL CONVENTION, MIPRO, p. $827-832,2010$.

SOOKHANAPHIBAM, K.; THAWONMAS, R. A Content Management System for User-Driven Museums. In: Second Life. In: INTERNATIONAL CONFERENCE ON CYBERWORDS (CW). p. 185189., 2009.

ZEB, M. A.; FASLI, M. Adaptive user profiling for devianting user interests. In: 3RD COMPUTER SCIENCE AND ELECTRONIC ENGINEERING CONFERENCE (CEEC). p. 65-70, 2011. 